\title{
Accelerated and increased joint damage in young mice with global inactivation of mitogen-inducible gene 6 after ligament and meniscus injury
}

\author{
Danese M Joiner ${ }^{1,4^{*}}$, Kennen D Less ${ }^{1}$, Emily M Van Wieren ${ }^{1}$, Yu-Wen Zhang ${ }^{2}$, Daniel Hess ${ }^{3}$ and Bart O Williams ${ }^{1}$
}

\begin{abstract}
Introduction: Ligament and meniscal damage can cause joint disease. Arthritic joints contain increased amounts of epidermal growth factor receptor (EGFR) protein, and polymorphisms in EGFR are associated with arthritis risk. The role of endogenous EGFR regulation during joint disease due to ligament and meniscal trauma is unknown. Mitogen-inducible gene 6 (MIG-6) can reduce EGFR phosphorylation and downstream signaling. We examined the effect of EGFR modulation by MIG-6 on joint disease development after ligament and meniscus injury.
\end{abstract}

Methods: Knee ligament transection and meniscus removal were performed surgically on mice homozygous for a global inactivating mutation in MIG-6 $\left(\mathrm{Mig}^{-6^{-1}}\right)$ and in wild-type (WT) animals.

Results: Two weeks after surgery, Mig- $6^{-1-}$ mice had bone erosion as well as greater fibrous tissue area and serum RANKL concentration than WT mice. Four weeks after surgery, Mig- $6^{-1-}$ mice had less cartilage and increased cell proliferation relative to contralateral control and WT knees. Increased apoptotic cells and growth outside the articulating region occurred in Mig- $6^{-1}$ mice. Tibia trabecular bone mineral density (BMD) and the number of trabeculae were lower in surgically treated knees relative to the respective control knees for both groups. BMD, as well as trabecular thickness and number, were lower in surgically treated knees from Mig- $6^{-/}$mice relative to WT surgically treated knees. Phosphorylated EGFR staining in surgically treated knees decreased for WT mice and increased for Mig- $6^{-1-}$ mice. Fewer inflammatory cells were present in the knees of WT mice.

Conclusion: Mig- $6^{-1-}$ mice have rapid and increased joint damage after ligament and meniscal trauma. Mig-6 modification could lessen degenerative disease development after this type of injury.

\section{Introduction}

Degenerative joint disease is painful and debilitating for millions of individuals. Such disease is typically classified as osteoarthritis (OA) or rheumatoid arthritis (RA). OA is characterized by progressive loss of articular cartilage, formation of bony outgrowths (osteophytes) and loss of mobility [1]. During OA, the extracellular matrix (ECM) network of cartilage tissue degrades, water content increases, the size of ECM molecules decreases, the structure of the collagen network is damaged and chondrocytes attempt to compensate through increased

\footnotetext{
* Correspondence: speededj@med.umich.edu

${ }^{1}$ Center for Skeletal Disease and Tumor Metastasis, Van Andel Research Institute, 333 Bostwick Avenue NE, Grand Rapids, MI 49503, USA

${ }^{4}$ Division of Pulmonary \& Critical Care Medicine, University of Michigan, Biomedical Science Research Building, 109 Zina Pitcher Place Room 4062, Ann Arbor, Ml 48109-2200, USA

Full list of author information is available at the end of the article
}

proliferation and metabolism [2]. OA chondrocytes proliferate and cluster as well as increase the production of both matrix proteins and matrix-degrading enzymes [3]. Decreased chondrocyte metabolism and an inability to adequately repair and regenerate the damaged tissue lead to complete loss of cartilage tissue, subchondral bone fibrillation and cyst formation $[2,4,5]$.

The results of work by other researchers also suggest that ECM mutations and disruption, as well as alterations in the mechanotransductory sensory feedback loop, which translates extracellular information to the cell to facilitate the secretion of ECM, may contribute to OA pathogenesis [6,7]. Pathologic cellular and structural changes in ligaments, the supporting musculature and fibrocartilaginous structures such as the meniscus and the synovium (that is, synovial lining hyperplasia, macrophage and lymphocyte infiltration, neoangiogenesis, and 
fibrosis) are also observed in OA [8]. RA is characterized by the presence of an immune-mediated inflammatory synovitis that can invade and destroy the ECMs of joint cartilage and bone [9]. Currently, there is no preventative or regenerative therapy for degenerative joint disease and in an effort to advance treatment, research has been focused on identifying the cell signaling pathways involved in arthritic disease development [3].

The epidermal growth factor receptor (EGFR) signaling pathway plays important roles in postnatal somatic growth, chondrocyte differentiation and bone metabolism [10-14]. EGFR is a tyrosine kinase receptor that binds to a family of epidermal growth factor (EGF)like ligands, including EGF, amphiregulin, transforming growth factor $\alpha$ (TGF $\alpha$ ), heparin-binding EGF (HBEGF), betacellulin (BTC) and epiregulin. Upon ligand binding, EGFR activates intracellular signal transduction pathways (including Ras/Raf/mitogen-activated protein kinase (MAPK) and phosphoinositide 3-kinase (PI3K)/ Akt) in a variety of cells, thus modulating proliferation, survival, adhesion, migration and differentiation [11].

Mice with osteoblast-specific deletion of EGFR have a low bone mass phenotype, whereas mice with high EGFR activity have a high bone mass phenotype [13]. Inhibited EGFR signaling can alter endochondral ossification in mice and rats, and EGFR global knockout (KO) mice display increased chondrocyte hypertrophy $[14,15]$. TGF $\alpha$ signaling controls endochondral ossification during bone growth [16]. EGFR signaling is involved in diseases that affect cartilage. In humans, there is a significant increase in the risk of RA development with the occurrence of the single-nucleotide polymorphism rs17337023 in EGFR, and arthritic joints have increased EGFR2 protein in the synovial fluid and membrane $[17,18]$. Pharmacological inhibition of EGFR can reduce the severity of collagen-induced RA in mice, and the inhibition of EGFR by adenovirus can reduce joint damage in arthritic mice $[19,20]$. Increased TGF $\alpha$ and EGFR signaling have also been shown in rat OA [21,22].

Little is known about the influence of endogenous EGFR regulation on skeletal diseases. Exogenous biglycan can regulate the expression of EGFR in human chondrocytes, and mitogen-inducible gene 6 (MIG-6) also known as Gene 33, ErbB receptor feedback inhibitor 1 (ERRFI1) and RALT-is a negative feedback regulator of EGFR [23,24]. The MIG-6 protein is a nonkinase scaffolding adaptor found in the cytosol. When transcriptionally induced by EGF, MIG-6 can reduce EGFR tyrosine phosphorylation and thus also reduce downstream extracellular signal-regulated kinase (ERK), c-Jun $\mathrm{N}$-terminal kinase (JNK) and protein kinase B (Akt) activation through direct interaction with the EGFR family [24,25]. MIG-6 is an immediate early response gene whose expression can be rapidly and robustly induced under normal and pathological conditions by hormones, growth factors and stress [24]. Reports of downregulated MIG-6 expression in a rat surgical meniscectomy OA knee model and spontaneous development of an OA-like phenotype in 3-month-old mice with a global Mig-6 KO inactivating mutation $\left(\mathrm{Mig}^{-6^{-/-}}\right)$ suggest a role for MIG-6 in joint disease. The influence of endogenous EGFR regulation on joint disease progression after trauma to ligaments and menisci has been unexplored [26,27].

Ligament and meniscus injury can alter mechanical loads placed on the joint and cause severe damage to the osteochondral joint surface and underlying trabecular bone, thus contributing to the onset and progression of joint disease [28-30]. We used a ligament/meniscus transection model in Mig- $6^{-1}$ mice to investigate the endogenous regulation of EGFR by MIG-6 and its effects on cartilage and bone damage after severe trauma. The identification of early signaling cascades and knowledge of cell behavior after ligament and meniscus injury are vital to developing therapies for subsequent arthritis development and progression. Therefore, we examined the early effects of ligament and meniscus injury on bone and cartilage in $\mathrm{Mig}^{-6^{-/}}$and wild-type (WT) mice. We hypothesized that global KO of Mig-6 in mice would increase EGFR signaling and disrupt cartilage and subchondral bone homeostasis, leading to severe damage to the cartilage and subchondral bone after ligament and meniscus injury. Furthermore, we sought to determine whether untreated ligament and meniscus injury would rapidly progress to severe degenerative joint disease in Mig- $6^{-/-}$mice.

\section{Materials and methods Ethical approval}

The Van Andel Research Institute Institutional Animal Care and Use Committee approved all experimental procedures.

Generation of mice and surgical destabilization model

Mig- $6^{-1-}$ mice were generated as described previously [26]. The percentage of Mig- $6^{-1-}$ mice alive at birth was less than that of heterozygous and WT littermates $(6 \%$ of Mig- $6^{-/-}, 65 \%$ of Mig- $6^{+/-}$and $29 \%$ of WT mice); therefore, some $M i g-6^{-/}$sample groups were smaller than the corresponding WT groups for experimental assays [31]. Transection of the cruciate and meniscotibial ligaments, as well as meniscus removal, were performed on the right knees of 4-week-old Mig- $6^{-/}$mice $(n=36)$ and their 4-week-old WT littermates $(n=42)$ using an adaptation of a previously described surgical method [32].

Mice were anesthetized by intraperitoneal injection of $0.3 \mathrm{ml} / \mathrm{g}$ tribromoethanol and received $0.1 \mathrm{ml}$ of ketoprofen. The right medial meniscotibial ligament was 
sectioned to destabilize the medial meniscus. The anterior cruciate, posterior cruciate and lateral meniscotibial ligaments were transected, and the medial and lateral menisci were removed. The patellar tendon and collateral ligaments were not transected. The incision was then closed with sutures and stapled. The animals resumed normal ambulation within 1 - 2 hours after surgery. Mig- $6^{-/}$mice showed spontaneous joint space narrowing at 1.5 months of age and increased joint damage severity at 3 months of age. Therefore, surgery was performed prior to spontaneous disease onset on 4-week-old mice. Mice were killed either 2 or 4 weeks after surgery by $\mathrm{CO}_{2}$ inhalation for endpoint analyses to examine the influence of injury prior to observation of significant spontaneous disease. On the basis of the limited number of $M i g-6^{-1-}$ mice per litter and the survival rate of Mig- $6^{-/-}$animals after surgery, we used the left knee from each animal as an untouched contralateral control to acquire the sample sizes we needed for the outcome measures. Other investigators have found negligible cartilage damage similar to WT age-matched controls in the contralateral joints of mice subjected to surgical destabilization [33].

\section{In vivo X-ray and micro-computed tomography}

WT $(n=3)$ and Mig- $6^{-/-}(n=3)$ mice were anesthetized with $2.5 \%$ isoflurane in oxygen and X-rayed every week after surgery with a piXarray digital specimen radiography system (Bioptics Faxitron, Tucson, AZ, USA). Knees from WT mice $(n=10)$ and $M i g-6^{-1}$ mice $(n=6)$ were imaged after excision at 28 days after surgery using a SKYSCAN 1172 micro-CT scanner (Bruker, Ettlingen, Germany). Samples were immersed in phosphate-buffered saline and scanned, and damage to the knee joint trabecular bone was analyzed according to established laboratory protocols [34].

\section{Histology}

Knees from WT mice $(n=17)$ and $M i g-6^{-/-}$mice $(n=14)$ were fixed and decalcified. Paraffin-embedded sections $(5 \mu \mathrm{m})$ were taken from the center of the medial condyle through the center of the lateral condyle. Sagittal sections were stained with hematoxylin and eosin and Movat's pentachrome according to standard laboratory protocols in order to examine cartilage and subchondral bone. Nine sections from each sample were used for each stain. The greatest perpendicular distance of articular cartilage thickness was measured with ImageJ software (US National Institutes of Health, Bethesda, MD, USA). Three physicians blinded to the samples scored histological sections according to the Osteoarthritis Research Society International scoring scale [35]. The lowest score of 0 on this scale corresponds to normal tissue and the highest score of 6 corresponds to bone remodeling and microfibrocartilaginous/osseous repair. The three grades for each section were averaged, and the data from each group of mice were combined.

EGFR signaling is important for osteoclastogenesis; therefore, tartrate-resistant acid phosphatase (TRAP) staining was performed with a kit obtained from SigmaAldrich (St Louis, MO, USA) according to manufacturer's instructions to detect osteoclasts [36]. A terminal deoxynucleotidyl transferase deoxyuridine triphosphate nick-end labeling (TUNEL) assay was performed according to the manufacturer's instructions to assess cellular apoptosis (Promega, Madison, WI, USA). Immunohistochemistry (IHC) analysis was performed with a VECTASTAIN ABC Kit (Vector Laboratories, Burlingame, CA, USA) for Ki67 (SB M3060 at 1:100 dilution), a marker for cell proliferation. We also performed IHC on sections for monocytes, neutrophils and macrophages (prediluted ab75693; the immunogen for this product is an affinity-purified monocyte membrane preparation) according to the manufacturer's instructions. IHC negative controls were incubated in blocking buffer instead of primary antibody. Stains were imaged with a Nikon Eclipse 55i microscope equipped with a Nikon Digital Sight camera (Nikon Instruments, Melville, NY, USA). The percentage of pixels that stained positive for Ki67, as well as monocytes, neutrophils and macrophages, was quantified using a Nuance system (Burlington, MA, USA). Quantitative stain analysis was performed on sections taken from the same-depth regions of the knee joints in WT and $\mathrm{KO}$ mouse control and surgically treated knees. Immunofluorescence (IF) was performed on sections for phospho-EGFR (1:800) and total EGFR (1:50) according to the manufacturer's instructions. IF images were obtained using an EVOS system (Advanced Microscopy Group, Bothell, WA, USA), and all antibodies were purchased from Abcam (Cambridge, MA, USA), Cell Signaling Technology (Beverly, MA, USA), Spring Bioscience (Pleasanton, CA, USA) or EMD Millipore (Billerica, MA, USA).

\section{Quantitative RT-PCR}

Knees from WT mice $(n=8)$ and $M i g-6^{-/-}$mice $(n=7)$ were dissected under RNase-free conditions and homogenized in $1 \mathrm{ml}$ of TRIzol reagent (Life Technologies, Carlsbad, CA, USA) with a Fast Prep-24 tissue and cell homogenizer (MP Biomedicals, Solon, OH, USA). RNA was chloroform-precipitated and extracted with an RNeasy Mini Kit (QIAGEN, Valencia, CA, USA) according to the manufacturer's instructions. RNA concentration was calculated and integrity was analyzed using a NanoDrop 2000 spectrophotometer (Thermo Scientific, Wilmington, DE, USA) and an Agilent 2100 Bioanalyzer (Agilent Technologies, Santa Clara, CA, USA), respectively, and potential DNA contamination was removed by 
RNase-free DNase treatment (amplification grade DNaseI; Sigma-Aldrich). cDNA was made with a RevertAid First Strand cDNA Synthesis Kit (Fermentas, Glen Burnie, MD, USA) on $0.5 \mu \mathrm{g}$ of RNA. Primers used for markers of osteogenesis and chondrogenesis (Integrated DNA Technologies, Coralville, IA, USA) were as follows: sox9: 5' - GCAGACCAGTACCCGCATCT-3' and 5' -CTC GTTCAGCAGCCTCCAG-3'; aggrecan: 5' - AGGACCT GGTAGTGCGAGTG-3' and 5'-GCGTGTGGCGAAG AA-3'; col2a1: 5' - AATGGGCAGAGGTATAAAGATAA GGA-3' and 5'-CATTCCCAGTGTCACACACACA-3'; col10a1: 5' -TTCATCCCATACGCCATAAAG-3' and 5'-AGCTGGGCCAATATCTCCTT-3'; colla1: 5'-CCTG AGTCAGCAGATTGAGAACA-3' and 5'-CCAGTACTC TCCGCTCTTCCA-3', runx2: 5' -CTGCAAGAAGGCTC TGGCGT-3' and 5' -CGGTTGGTCTCGGTGGCTGG-3'; opn: 5'-GGCATTGCCTCCTCCCTC-3' and 5'-GCAGG CTGTAAAGCTTCTCC-3'; os $x$ : 5'-GCAACTGGCTAG GTGGTGGTC-3' and 5'-GCAAAGTCAGATGGGTAA GTAGGC-3'; ocn-1: 5' -CTCTGCTGACCCTGGCTG CG-3' and 5'-TGAGGCTCCAAGGTAGCGCC-3'; ocn-2: 5' - TGACCTCACAGATGCCAAGCCCA-3' and 5' -AGG GCTCTGGCCACTTACCCA-3'; and $18 s$ : 5'-GTAACC CGTTGAACCCCATT-3' and 5' -CCATCCAATCGGTA GTAGCG-3'.

\section{Western blot analysis}

Knees from WT mice $(n=14)$ and $M i g-6^{-/}$mice $(n=12)$ were homogenized in $1 \mathrm{ml}$ of radioimmunoprecipitation assay lysis buffer $(50 \mathrm{mM}$ Tris- $\mathrm{HCl}, 150 \mathrm{mM} \mathrm{NaCl}$, $1 \mathrm{mM}$ ethylenediaminetetraacetic acid, $1 \mathrm{mM}$ ethylene glycol tetraacetic acid, $2 \mathrm{mM}$ sodium orthovanadate, 20 nM sodium pyrophosphate, $1 \%$ Triton X-100, 1\% sodium deoxycholate, and $0.1 \%$ SDS) using a Fast Prep-24 tissue and cell homogenizer. Protein concentration was determined by bicinchoninic acid assay (Thermo Scientific, Rockford, IL, USA). Protein $(40 \mu \mathrm{g})$ was loaded into the lanes of a $4 \%$ to $20 \%$ Mini-PROTEAN TGX Precast Gel (Bio-Rad Laboratories, Hercules, CA, USA) and subjected to electrophoresis. Membranes were probed for phospho-EGFR, phospho-p44/42 ERK (pERK), total p44/42 ERK or $\beta$-tubulin.

\section{Enzyme-linked immunosorbent assay}

We measured receptor activator of the nuclear factor $\mathrm{kB}$ ligand (RANKL) and osteoprotegerin (OPG), which play important roles in the formation of active osteoclasts and can inhibit the RANKL/RANK interaction, respectively [37]. Blood $(200 \mu \mathrm{l})$ was collected from the orbital sinus of WT mice $(n=7)$ and Mig- $6^{-/-}$mice $(n=5)$ before surgery (baseline) and 2 and 4 weeks after ligament and meniscus injury according to established animal protocols. Blood was incubated for 2 hours at $37^{\circ} \mathrm{C}$ and spun down at $12,000 \mathrm{rpm}$ for 10 minutes at $4^{\circ} \mathrm{C}$, and serum was collected. OPG and RANKL concentrations were determined using enzyme-linked immunosorbent assay (ELISA) kits from R\&D Systems (Minneapolis, MN, USA) according to the manufacturer's instructions. All samples were assayed in duplicate.

\section{Statistical analysis}

GraphPad Prism software (GraphPad Software, La Jolla, CA, USA) was used for statistical analysis. We confirmed normality of the data sets and used a two-sided unpaired Student's $t$-test with Welch's correction for quantitative RT-PCR data. Repeated-measures analysis was used for ELISA data. Two-way analysis of variance with Tukey's test for multiple comparisons was used for all other quantitative data. We conducted an F-test to determine whether variances were significantly different between data sets and performed log transformation on data for which variances were significantly different. $P$-values less than 0.05 were considered statistically significant.

\section{Results}

\section{Early and severe degeneration of the knee joint after injury in Mig- $\mathbf{6}^{-/-}$mice}

Two weeks after ligament and meniscal transections, hyperplastic disorganized fibrous tissue formed in the knee joint space of Mig- $6^{-/-}$and WT mice. We observed a significantly greater fibrous tissue area and greater staining for fibrin in surgically treated knees of $M i g-6^{-/}$mice $(P<0.001)$ and WT mice $(P<0.05)$ relative to respective control knees (Figure 1A and $1 \mathrm{~B}$ ) The area of fibrous tissue in $M i g-6^{-/-}$surgically treated knees was significantly greater than that in WT control $(P<0.001)$ or surgically treated knees $(P<0.05)$ (Figure $1 \mathrm{~A}$ and $1 \mathrm{~B})$.

Four weeks after surgery, the hyperplastic fibrous tissue region was significantly greater in the surgically treated knees of Mig- $6^{-/}$mice $(P<0.001)$ and WT mice $(P<0.01)$ than in the knees of respective control mice (Figure $1 C$ and 1D). Surgically treated knees of $\mathrm{Mig}-6^{-/}$mice included cartilaginous pockets in areas outside the articulating surface (Figure 1C). We also qualitatively observed cartilage degradation in surgically treated knees from Mig- $6^{-/-}$mice and joint space narrowing in surgically treated knees from WT animals (Figure 1D). The fibrous tissue area was significantly greater for both knees from Mig- $6^{-/}$mice relative to the respective knees from WT animals $(P<0.0001)$ (Figure $1 C$ and $1 D)$.

Four weeks after surgery, joint morphology was qualitatively similar between male and female mice of each genotype (Additional file 1: Figure S1A). Therefore, samples were pooled for all quantitative analyses. Articular cartilage thickness was significantly lower in $\mathrm{Mig}^{-6^{-/-}}$ $(P<0.001)$ and WT $(P<0.05)$ surgically treated knees relative to respective control knees (WT control $=0.265 \pm$ 


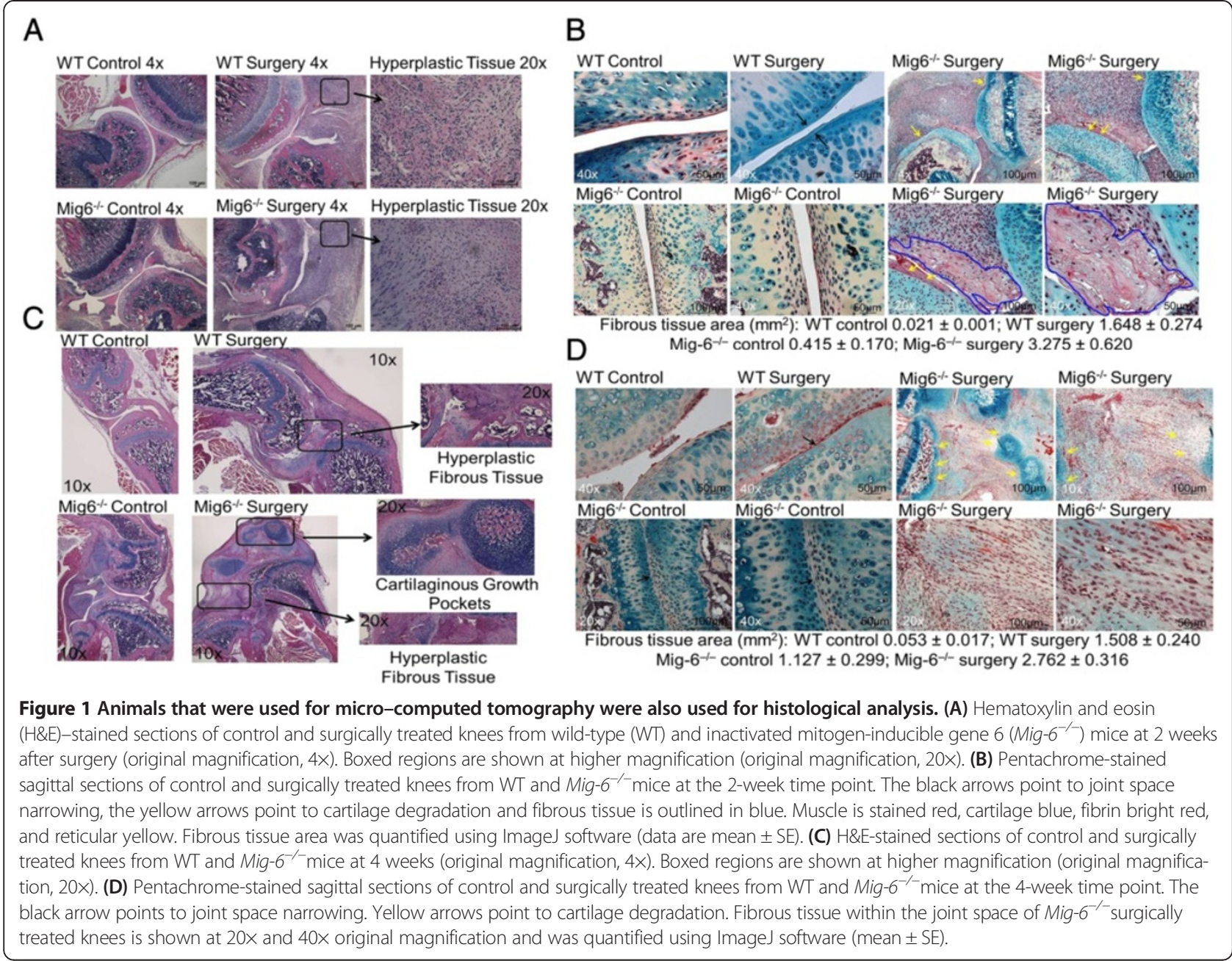

$0.023 \mathrm{~mm}$, WT surgically treated $=0.187 \pm 0.015 \mathrm{~mm}$, Mig- $6^{-/-}$control $=0.236 \pm 0.031 \mathrm{~mm}$ and Mig- $^{-6^{-1}}=$ surgically treated $=0.100 \pm 0.027 \mathrm{~mm}$ ). Articular cartilage thickness in surgically treated knees from $\mathrm{Mig}^{-6^{-/}}$mice was significantly lower than that in WT surgically treated mice $(P<0.05)$ and WT control mice knees $(P<0.0001)$. The histological score, indicative of disease severity, increased significantly with surgery for both $M i g-6^{-/}$mice $(P<0.01)$ and WT mice $(P<0.01)$ and was significantly higher for Mig- $6^{-/}$knees than for WT control knees $(P<0.05)$ (WT control $=1.1 \pm 0.3$, WT surgically treated $=4.8 \pm 0.3$, Mig- $6^{-/-}$control $=2.4 \pm 0.6$ and Mig- $6^{-/}$surgically treated $=5.3 \pm 0.3)$.

Cell proliferation and apoptosis were affected by injury and loss of Mig-6. There was a significant increase $(P<$ $0.001)$ in the percentage of Ki-67-stained pixels, indicative of cell proliferation, in $\mathrm{Mig}-\mathrm{6}^{-/-}$surgically treated knees relative contralateral control knees (Figure $2 \mathrm{~A}$ ). The percentage of proliferating cells was significantly greater $(P<$ $0.05)$ in surgically treated knees from $\mathrm{Mig}-6^{-1-}$ mice than in surgically treated knees from WT animals (Figure 2A).
Mig- $6^{-1-}$ mice had a significant increase $(P<0.01)$ in the area of the TUNEL staining in surgically treated knees relative to control knees, but there was no difference between the genotypes (Figure 2B).

We observed differences in chondrogenic and osteogenic markers in knees from Mig- $6^{-/-}$and WT mice. Of chondrogenic and osteogenic markers in $\mathrm{Mig}-\mathrm{6}^{-/}$surgically treated knees (relative to control knees), Sox9, Col10a1, Runx2, Opn and Ocn-2 were downregulated 5-, 1.8-, 1.9-, 2.1- and 2-fold, respectively, relative to controls (Figure 2C). In WT mice, Col10a1, Col1a1 and Ocn-2 were upregulated 1.9-, 2.8- and 1.7-fold, respectively, and Opn was downregulated 2.3-fold (Figure 2C). Relative Sox9 expression was significantly lower in Mig- $6^{-1-}$ knees than in WT $(P<0.01)$. Expression of Col10a1 $(P<0.01)$, Colla1 $(P<0.05)$ and Ocn-2 $(P<$ 0.01 ) was significantly lower as well (Figure $2 \mathrm{C}$ ).

\section{Changes in bone structure after injury and loss of Mig- 6}

Femoral condyle bone erosion was evident by X-ray 2 weeks after surgery in Mig- $6^{-/-}$mice, and deterioration 


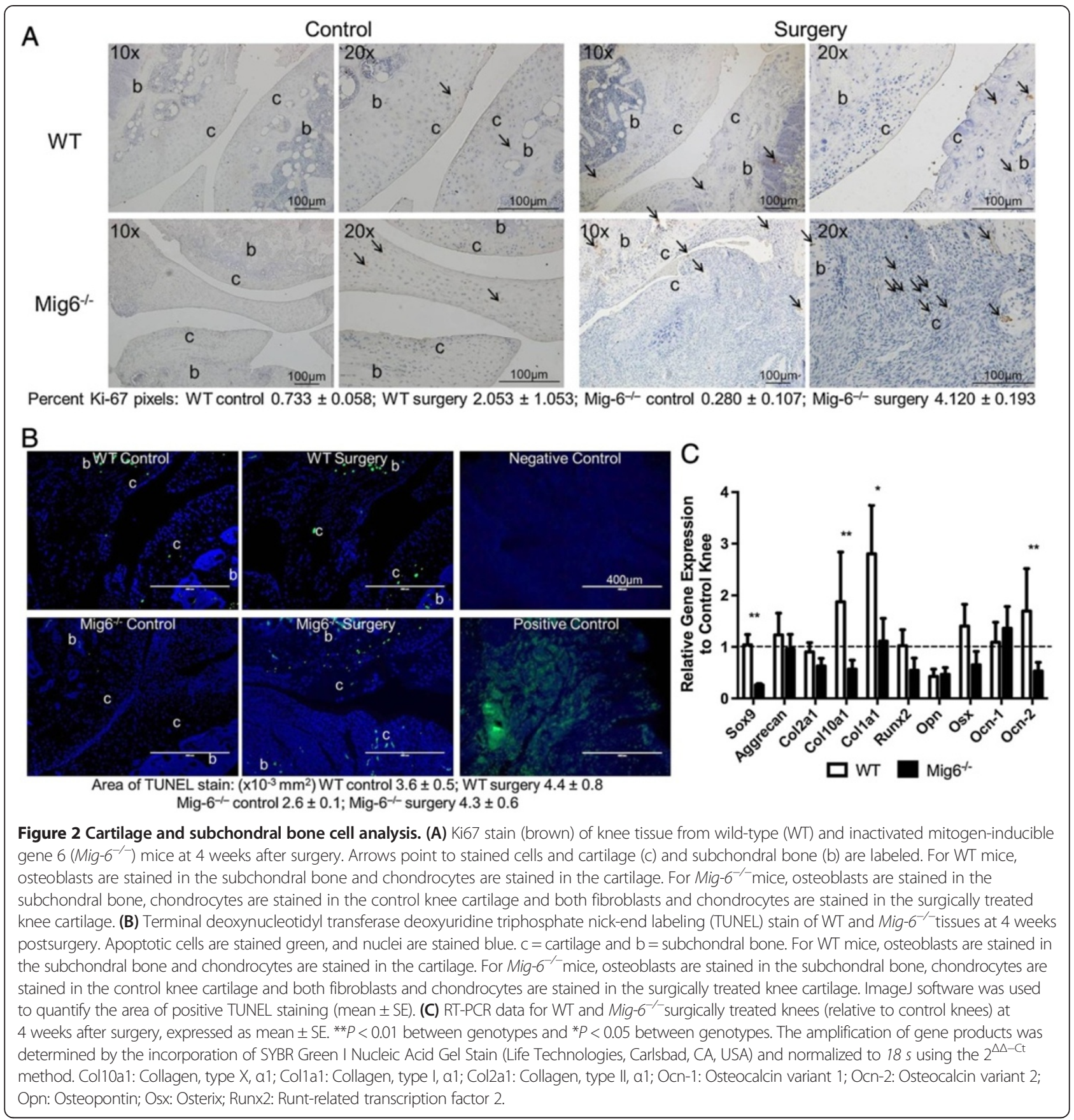

of the femoral subchondral bone increased in severity qualitatively at 4 weeks (Figure 3A). Subchondral bone damage was qualitatively observed in three-dimensional, reconstructed micro-CT images of surgically treated knees from $M i g-6^{-1-}$ mice at 4 weeks (Figure 3B). Relative to WT control knees, we observed differences in trabecular bone geometry in the distal femur and proximal tibia of WT surgically treated knees and in knees from Mig- $6^{-/-}$mice (Figure 3C and 3D).

In surgically treated knees relative to control knees, tibial trabecular bone density and the number of trabeculae decreased significantly $(P<0.05)$, but the separation distance between trabeculae increased significantly $(P<$ 0.01 ), for $M i g-6^{-1-}$ mice (Table 1). The thickness of trabeculae in the distal femur $(P<0.05)$ and proximal tibia $(P<0.01)$ were significantly higher in surgically treated knees of $M i g-6^{-1-}$ mice than in control knees, and the distal femur bone volume fraction (ratio of the segmented bone volume to the total volume of the region of interest) was significantly lower in WT surgically treated knees than in control knees $(P<0.05)$ (Table 1$)$. In the proximal tibia of $M i g-6^{-/}$surgically treated knees, 


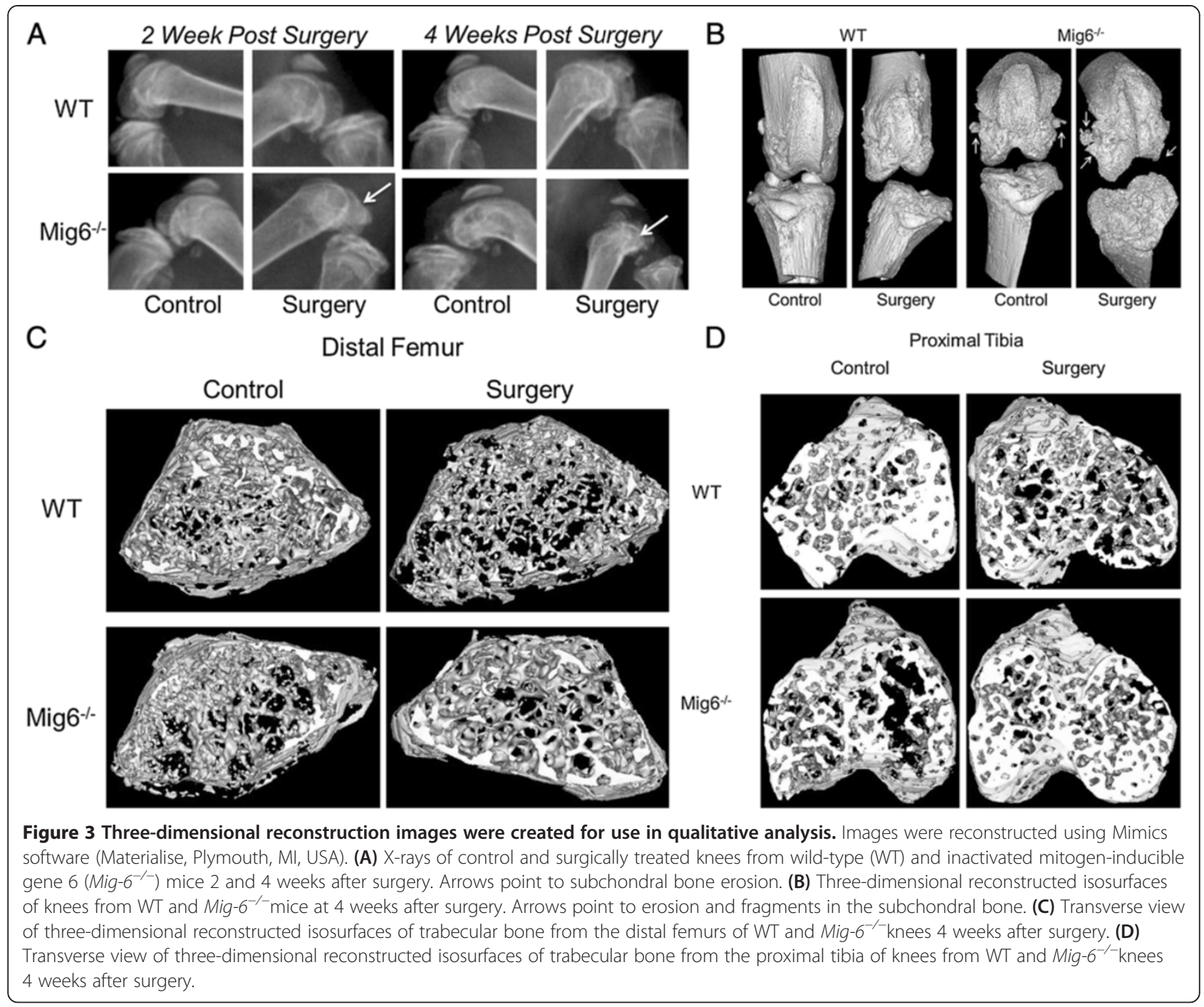

trabecular thickness $(P<0.001)$, trabecular BMD $(P<$ $0.01)$ and number of trabeculae $(P<0.05)$ were significantly lower than in the WT surgically treated knees (Table 1). Femur trabecular thickness was significantly higher in the surgically treated knees of $M i g-6^{-/}$mice than in WT surgically treated knees $(P<0.001)$ (Table 1$)$. Both tibia trabecular BMD $(P<0.05)$ and the number of femoral trabeculae $(P<0.01)$ were significantly lower in control knees of Mig- $6^{-/-}$mice than in WT control knees, and the distance between trabeculae was significantly larger for control knees of Mig- $6^{-/-}$mice than for WT control knees in this region $(P<0.05)$ (Table 1$)$. The effect of surgery on trabecular thickness within the tibia and femur was significantly different between genotypes $(P<$ 0.01 for interaction).

Increased inflammatory cells and osteoclasts in $\mathrm{Mig}^{-6^{-/}}$mice Serum RANKL concentration was significantly greater in $M i g-6^{-/}$mice 2 weeks after surgery relative to the concentrations in $\mathrm{Mig}-6^{-/-}$mice at baseline and at 4 weeks after surgery and relative to the concentration for WT animals at 2 weeks after surgery $(P<0.05)$ (Figure $4 \mathrm{~A})$. The effects of genotype on RANKL concentration over time were significantly different $(P<0.05$ for interaction). For WT mice, OPG concentration was significantly lower $(P<0.001)$ at 4 weeks after surgery relative to the concentrations at 2 weeks after surgery and at baseline (Figure 4B). Serum OPG concentration was significantly greater for $M i g-6^{-/}$mice at baseline $(P<0.001)$ and at 2 weeks after surgery $(P<0.001)$ and at 4 weeks after surgery $(P<0.0001)$ relative to the concentrations for WT animals (Figure $4 \mathrm{~B}$ ), but there were no significant differences in the RANKL/OPG ratios between genotypes or time points (Additional file 1: Figure S1B).

We observed significantly greater TRAP-positive cells normalized to tissue area in the subchondral bone of surgically treated knees from $\mathrm{Mig}-6^{-/-}$mice at 2 weeks relative to $\mathrm{Mig}-6^{-/-}$control knees $(P<0.01)$ and relative 
Table 1 Tibia and femur trabecular bone micro-CT data ${ }^{a}$

\begin{tabular}{|c|c|c|c|c|}
\hline \multirow[t]{2}{*}{ Bone } & \multicolumn{2}{|c|}{ WT } & \multicolumn{2}{|c|}{$M^{M i g}-6^{-/-}$} \\
\hline & Control & Surgery & Control & Surgery \\
\hline \multicolumn{5}{|l|}{ Tibia } \\
\hline Trabecular BMD (g/ml) & $0.579 \pm 0.010$ & $0.528 \pm 0.006^{b}$ & $0.524 \pm 0.028^{c}$ & $0.459 \pm 0.009^{b, d}$ \\
\hline Trabecular thickness (mm) & $0.022 \pm 0.000$ & $0.022 \pm 0.001$ & $0.020 \pm 0.001$ & $0.021 \pm 0.001^{e, f}$ \\
\hline Number of trabeculae & $5.918 \pm 0.280$ & $5.437 \pm 0.405^{b}$ & $4.395 \pm 0.744$ & $2.545 \pm 0.187^{g, h}$ \\
\hline Separation distance between trabeculae (mm) & $0.138 \pm 0.012$ & $0.139 \pm 0.004$ & $0.242 \pm 0.044$ & $0.289 \pm 0.063^{e}$ \\
\hline BV/TV (\%) & $12.473 \pm 0.483$ & $13.042 \pm 0.340$ & $8.385 \pm 1.780$ & $10.670 \pm 3.765$ \\
\hline \multicolumn{5}{|l|}{ Femur } \\
\hline Trabecular BMD (g/ml) & $0.590 \pm 0.011$ & $0.579 \pm 0.015$ & $0.551 \pm 0.019$ & $0.533 \pm 0.022$ \\
\hline Trabecular thickness (mm) & $0.025 \pm 0.001$ & $0.023 \pm 0.001$ & $0.024 \pm 0.002$ & $0.031 \pm 0.001^{b, f}$ \\
\hline Number of trabeculae & $6.214 \pm 0.632$ & $4.866 \pm 0.419$ & $3.716 \pm 0.093^{i}$ & $4.573 \pm 0.545$ \\
\hline Separation distance between trabeculae $(\mathrm{mm})$ & $0.124 \pm 0.009$ & $0.158 \pm 0.023$ & $0.225 \pm 0.040^{c}$ & $0.190 \pm 0.033$ \\
\hline BV/TV (\%) & $16.502 \pm 1.011$ & $10.893 \pm 1.069^{b}$ & $11.747 \pm 2.764$ & $14.499 \pm 2.678$ \\
\hline
\end{tabular}

${ }^{a} B M D$ : Bone mineral density; BV/TV: Bone volume fraction (ratio of the segmented bone volume to the total volume of the region of interest); Mig- ${ }^{-/-}$: Inactivated mitogen-inducible gene 6 ; WT: Wild type. Animals that were X-rayed were also used for micro-CT analysis. Data are expressed as mean \pm SE. ${ }^{b} P<0.05$ between control and surgery. ${ }^{c} P<0.05$ between genotypes for control knees. ${ }^{\mathrm{d}} P<0.01$ between genotypes for surgically treated knees. ${ }^{\mathrm{e}} P<0.01$ between control and surgery. ${ }^{f} P<0.001$ between genotypes for surgically treated knees. ${ }^{9} P<0.001$ between control and surgery. ${ }^{\text {h}} P<0.05$ between genotypes for surgically treated knees. ${ }^{i} P<0.01$ between genotypes for control knees.

to knees from WT animals $(P<0.001)$ (Figure $4 \mathrm{C})$. We found significantly greater TRAP-positive cells in the subchondral bone of control knees from $\mathrm{Mig}-6^{-/}$mice at 4 weeks relative to control knees from WT mice (Figure 4D). We did not find monocytes, neutrophils or macrophages in WT or Mig- $6^{-1-}$ mice 2 weeks after surgery (data not shown). The percentage of stained pixels for monocytes, neutrophils and macrophages was significantly greater in control and surgically treated knees of $M i g-6^{-1-}$ mice than in WT controls $(P<0.001$ and $P<0.01$, respectively) or WT surgically treated knees $(P<0.01$ and $P<0.01$, respectively) (Figure 5A).

\section{Differences in alteration of phosphorylated epidermal growth factor receptor protein after injury}

The area of phospho-EGFR staining was significantly lower at 4 weeks in control knees from $\mathrm{Mig}-6^{-/-}$mice relative to surgically treated knees $(P<0.01)$ (Figure 5B). The area of phospho-EGFR staining was significantly lower at 4 weeks in surgically treated knees from WT mice relative to control knees $(P<0.0001)$ and also to surgically treated knees from $\mathrm{Mig}-6^{-/}$mice $(P<0.05)$ (Figure $5 \mathrm{~B}$ ). The effect of surgery on phospho-EGFR staining in WT and Mig- $6^{-1-}$ mice at 4 weeks was significantly different $(P<0.0001$ for interaction).

There were no significant differences in the area of EGFR staining between control and surgically treated knees from WT and Mig- $6^{-/-}$mice at 4 weeks or between genotypes (Figure 5C). Phospho-EGFR densitometry was significantly lower at 4 weeks in surgically treated knees from WT mice relative to control knees $(P<0.05)$ and also to surgically treated knees from $\mathrm{Mig}-6^{-/-}$mice
$(P<0.0001)$ (Figure 5D). Phospho-EGFR densitometry was significantly lower at 4 weeks in control knees from Mig- $6^{-/-}$mice relative to surgically treated knees $(P<$ 0.0001 ) (Figure 5D). There were no significant differences in pERK densitometry or total ERK densitometry between control and surgically treated knees from WT and $\mathrm{Mig}-6^{-/-}$mice at 4 weeks or between genotypes (Figure 5D and Additional file 2: Table S1). The phosphoERK densitometry normalized to total ERK densitometry was significantly lower in control knees $(P<0.01)$ and surgically treated knees $(P<0.0001)$ from WT mice relative to control knees from $M i g-6^{-/}$mice (Figure 5D).

\section{Discussion}

We found exacerbated and accelerated cartilage and subchondral bone damage in young $M i g-6^{-/-}$mice after ligament and meniscal trauma, as well as increased EGFR phosphorylation. Increased cell proliferation has been reported in $M i g-6^{-1-}$ mice, and reduced proliferation has been reported in cells overexpressing Mig-6 and treated with hepatocyte growth factor [31,38]. We found no differences between genotypes in terms of control knee cell proliferation, but we observed a greater number of proliferating cells and staining for phospho-EGFR in surgically treated knees from Mig- $6^{-1-}$ mice (relative to their control knees and to surgically treated knees from WT mice), which likely contributes to increased cell growth. Despite increased proliferation, Sox 9 gene expression was downregulated in $M i g-6^{-1}$ surgically treated knees, which could be due to reduced articular cartilage.

We did not detect consistent increases in phosphoERK, total ERK or phospho-EGFR protein levels for both 


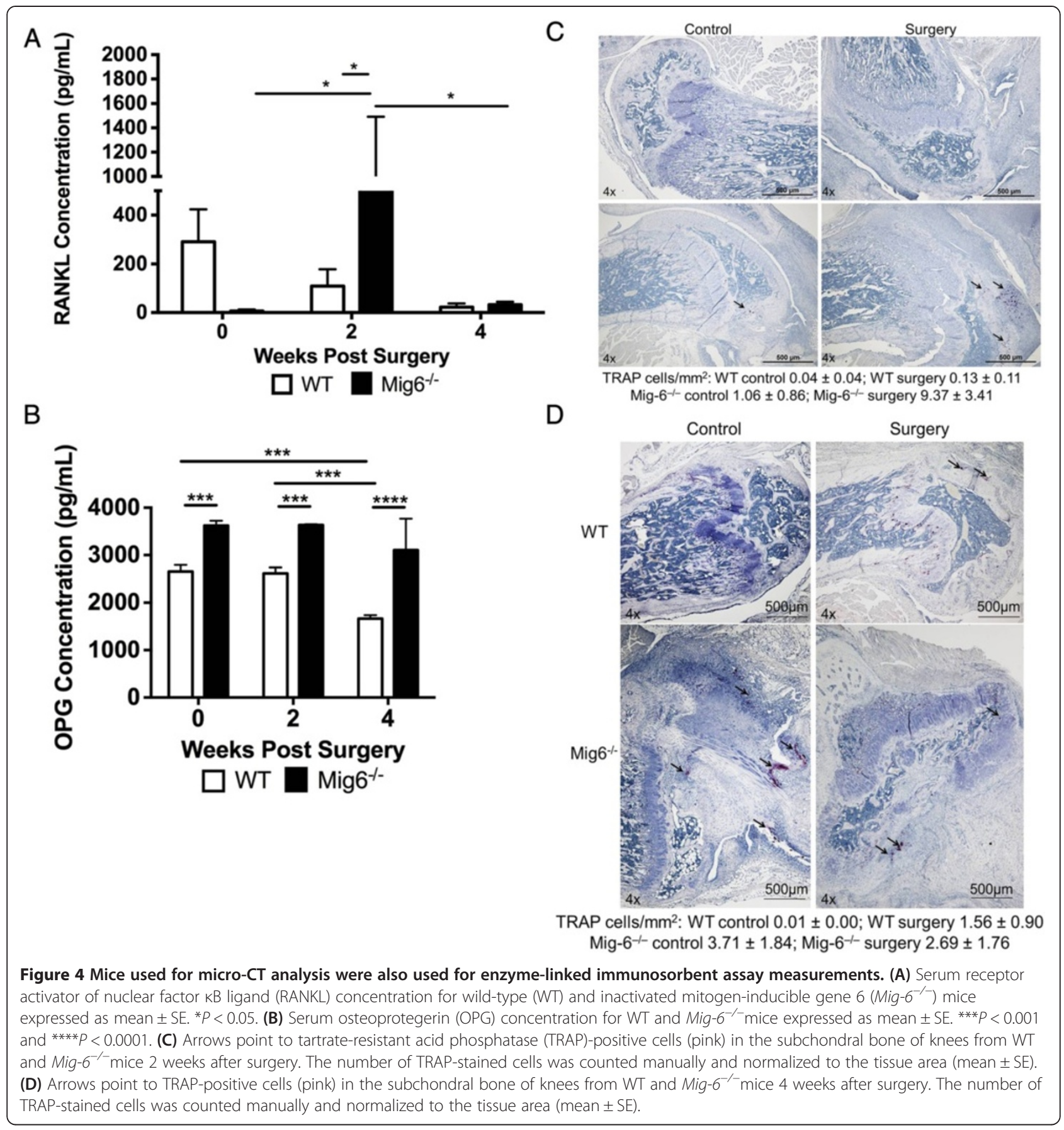

knees from $\mathrm{Mig}-6^{-/-}$mice relative to WT, but these proteins can be influenced by other pathways that balance EGFR signaling. EGFR signaling is maintained by the endocytic pathway, which is crucial for the spatial localization of the EGF-EGFR complex needed to activate downstream effectors [39]. EGF binding can cause the EGF-EGFR complex to internalize and traverse the endocytic pathway, moving from clathrin-coated vesicles through endosomes to lysosomes for degradation [39]. This process, though it was not examined in this study, could sequester EGFR from downstream effectors in addition to removing the receptor from the cell surface, dephosphorylating the receptor or targeting the ligandreceptor complex for degradation [39].

Investigators in several studies have highlighted the influence of Mig-6 and EGFR on apoptosis. Pharmacological compounds can induce apoptosis in lung and breast cancer cells by inhibiting the EGFR pathway, and recent work has demonstrated that Mig-6 actively senses EGF deprivation to directly activate proapoptotic c-Abl 


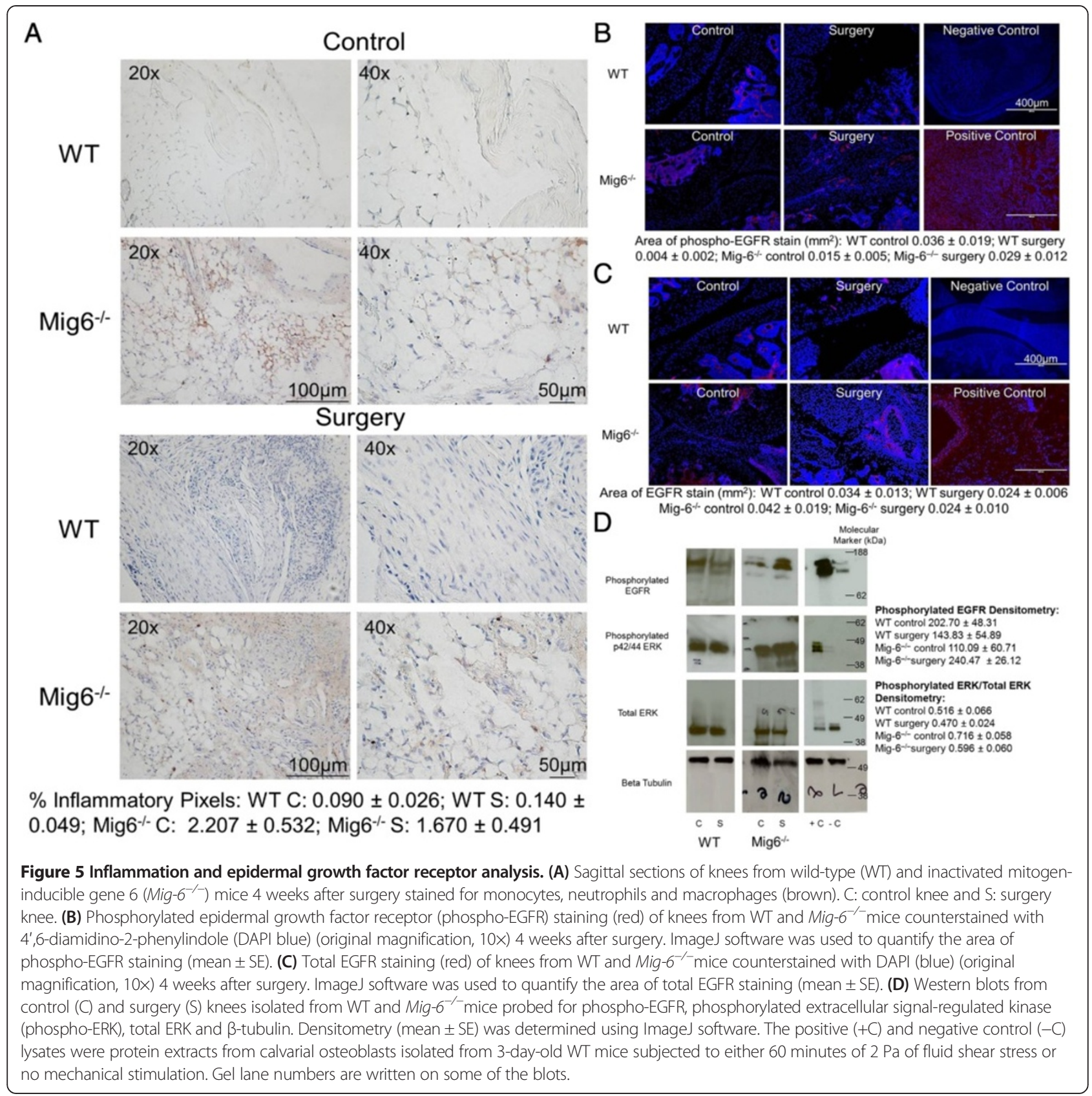

in murine epithelial cells [40-42]. Mig-6 can trigger apoptosis as well as exacerbate endoplasmic reticulum stress-induced $\beta$-cell death [43]. Apoptosis inhibition in MCF7 human breast cancer cells with exogenous overexpression of Mig-6 has been shown, but increased cleaved caspase 3 immunostaining in lungs from $\mathrm{Mig}-\mathrm{6}^{-/}$mice has also been reported $[31,44]$. In our present study, we observed an increase in apoptotic cells in the surgically treated knees from Mig- $6^{-/-}$mice relative to control knees, which may be attributed to increased EGFR phosphorylation and also to damage in the articular cartilage and underlying subchondral bone.
EGFR signaling has been linked to inflammation, but limited data exist regarding the effect of Mig-6 on inflammation. Histological reports show staining for EGFR in the bronchial epithelium, glands, smooth muscle cells and basement membranes of chronically inflamed asthmatic airways, and EGFR inhibition can reduce the number of inflammatory cells, as well as interleukin 4 and interleukin 13 concentrations, in bronchoalveolar lavage fluid $[45,46]$. EGFR inhibition can also reduce eosinophil recruitment in the lungs as well as airway hyperresponsiveness. The concentrations of serum and joint EGF and EGFR are significantly higher in RA patients than in 
healthy populations, and the thymuses of $M i g-6^{-1-}$ mice are larger than normal size; however, significant numbers of inflammatory cells were not observed in previous studies of Mig- $6^{-1-}$ joints [26,46]. Mig-6 can be transcriptionally induced by hormones and growth factors as well by stresses such as mechanical strain and impact $[47,48]$. In Mig- $6^{-1-}$ mice, we found increased numbers of inflammatory cells, which may occur naturally in younger Mig- $6^{-/}$mice prior to observation of significant spontaneous disease and could also be a compensatory response to the stresses placed on the surgically treated knee joint by the altered mechanical environment induced by ligament and meniscus injury [49].

Relative to WT animals, we observed some impairment to trabecular bone in the control and surgically treated knees of $\mathrm{Mig}-\mathrm{6}^{-/-}$mice, which could reduce the mechanical integrity of the knee joints [50]. There was an increase in trabecular thickness with surgery in Mig- $6^{-1-}$ mice, which may be a result of skeletal adaptation and an attempt to compensate for joint damage and/or increased EGFR phosphorylation [13]. There is also evidence that EGFR signaling is involved in osteoclast formation and that it may have functional importance for osteoclast differentiation and survival. Thus, increased EGFR phosphorylation in surgically treated knees of Mig- $6^{-/-}$mice could contribute to the observed subchondral bone resorption and increased RANKL [36].

It has been suggested that the impact of Mig- 6 might manifest only during dynamic processes such as development or injury repair [31]. EGFR is activated in response to a wounding event, and signaling pathways downstream from EGFR are central in regulating distinct stages of wound healing [51]. Together, the inactivation of Mig-6 and increased EGFR phosphorylation in surgically treated knees of mutant mice may increase inflammation and thus apoptosis, leading to reduced articular cartilage thickness. Reduced cartilage thickness in $M i g-6^{-1}$ mice could further increase the loads on the joint, causing additional damage to the underlying subchondral bone. Despite increased cell proliferation (perhaps in an enhanced attempt to repair damage), the joint integrity in Mig- $6^{-/-}$mice remains compromised because of undirected cell growth. Therefore, $M i g-6^{-/}$mice develop rapid and more severe cartilage and subchondral bone damage after ligament and meniscus injury.

Four weeks after surgery, we observed joint space narrowing in control knees from Mig- $6^{-/-}$mice that was comparable to that previously reported in 1.5 -month-old Mig- $6^{-/-}$animals, but we did not observe joint space narrowing in WT control knees and therefore attribute this phenomenon to early-stage joint disease associated with loss of function in Mig- $6^{-/-}$animals. Fifty percent of Mig- $6^{-/}$mice die before adolescence, likely because of impairments in pulmonary development and vascularization of the lungs [31]. The limited survival of adult Mig- $6^{-/}$mice enabled us to study only early changes to cartilage and subchondral bone after trauma to ligaments and the meniscus in young mice. Several cell types are found in the knee joint under normal and pathological conditions. Synovial lining cells produce lubricin and hyaluronic acid, which help to protect and maintain the integrity of the cartilage surface [8]. Fibroblasts, macrophages and lymphocytes participate in synovial hyperplasia and inflammation, and osteoblasts, osteoclasts and chondrocytes, respectively, form and resorb bone and maintain cartilage $[3,8,52]$. In our present study, we were able to observe the effect of inactivation of Mig-6 in all cell types involved in joint maintenance after ligament and meniscal damage, but we did not completely elucidate the exact mechanism of action of Mig-6 in joints. Our study opens the door for future work focused on dissecting the exact mechanism of Mig- 6 and EGFR signaling in joint damage after ligament and meniscus injury.

\section{Conclusion}

Phospho-EGFR was lower in surgically treated knees from WT mice relative to controls, and joint damage was less severe in these animals relative to $\mathrm{Mig}-6^{-1-}$ mice, which conversely had increased phospho-EGFR in surgically treated knees relative to controls. There could be a threshold of EGFR signaling required for maximum therapeutic effect after trauma to the ligaments and meniscus, and attenuation of Mig- 6 could be a strategic tool for finely altering EGFR signaling and reducing cartilage and subchondral bone damage after this type of injury.

\section{Additional files}

Additional file 1: Figure S1. (A) Pentachrome-stained sagittal sections of control and surgically treated knees from male and female wild-type (WT) and inactivated mitogen-inducible gene $6\left(\mathrm{Mig}^{-6^{-1}}\right)$ mice 4 weeks after surgery. Muscle (red), cartilage (blue), fibrin (bright red) and reticular fibers (yellow) are shown. Original magnification, 4x. (B) Serum RANKL (receptor activator of nuclear factor $\mathrm{KB}$ ligand) concentration normalized to osteoprotegerin concentration for WT and $\mathrm{Mig}^{-6^{-/}}$mice expressed as mean $\pm \mathrm{SE}$.

Additional file 2: Table S1. Western blot densitometry.

\section{Abbreviations}

Akt: Protein kinase B; ANOVA: Analysis of variance; BMD: Bone mineral density; BTC: Betacellulin; BV/TV: Bone volume fraction (ratio of the segmented bone volume to the total volume of the region of interest); Col10a1: Collagen type $X$, a1; Col1a1: Collagen type I, a1; Col2a1: Collagen type II, a1; DAPI: 4',6-diamidino2-phenylindole; ECM: Extracellular matrix; EDTA: Ethylenediaminetetraacetic acid; EGF: Epidermal growth factor; EGFR: Epidermal growth factor receptor; ELISA: Enzyme-linked immunosorbent assay; ER: Endoplasmic reticulum; ERK: Extracellular signal-regulated kinase ( $p 44 / 42$ mitogen-activated protein kinase); ERRFI1 and ERBB: receptor feedback inhibitor 1; HB-EGF: Heparin-binding epidermal growth factor; IF: Immunofluorescence; IHC: Immunohistochemistry; JNK: c-Jun N-terminal kinase; KO: Knockout; MAP kinase: Mitogen-activated protein kinase; MIG-6: Mitogen-inducible gene 6; NaCl: Sodium chloride; OA: Osteoarthritis; Ocn-1: Osteocalcin variant 1; Ocn-2: Osteocalcin variant 2; OPG: Osteoprotegerin; Opn: Osteopontin; Osx: Osterix; pERK and phospho- 
ERK: Phosphorylated extracellular signal-regulated kinase (phosphorylated p44/42 mitogen-activated protein kinase); Phospho-EGFR: Phosphorylated epidermal growth factor receptor; PI3kinase: Phosphatidylinositide 3-kinase; RA: Rheumatoid arthritis; RALT: Receptor-associated late transducer; RANK: Receptor activator of nuclear factor $\mathrm{KB}$; RANKL: Receptor activator of nuclear factor $\mathrm{KB}$ ligand; Runx2: Runt-related transcription factor 2; TFG-a: Transforming growth factor a; TRAP: Tartrate-resistant acid phosphatase; TUNEL: Terminal deoxynucleotidyl transferase deoxyuridine triphosphate nick-end labeling; WT: Wild type.

\section{Competing interests}

The authors declare that they have no competing interests.

\section{Authors' contributions}

$\mathrm{DMJ}, \mathrm{KDL}$, and BOW designed the research. DMJ, KDL, EMV and DH performed the research. DMJ, KDL and EMV analyzed the data. DMJ wrote the paper, which was edited by KDL, EMV, DH and BOW. All authors read and approved the final manuscript.

\section{Authors' information}

DMJ is a research fellow in internal medicine, pulmonary/critical care and immunology at the University of Michigan and a former fellow at the Laboratory of Cell Signaling and Carcinogenesis, Van Andel Research Institute. BOW is an associate professor and director of the Center for Skeletal Disease and Tumor Metastasis, as well as the head of the Laboratory of Cell Signaling and Carcinogenesis, at the Van Andel Research Institute.

\section{Acknowledgements}

The authors thank George Vande Woude, Marian Young, Frank Beier, Mike Morris, Martin Hoffmann, Kuldeep Gadkari, Donald Jay Scholten II, Cassie Diegel, Dominic Pell and Matt Steensma for their scientific contributions; Bree Berghuis, Lisa Turner, Kristin Feenstra, James Resau and Galen Hostetter for their assistance with histological experiments; Susan Budnick, Lisa Kefene and the vivarium staff at the Van Andel Institute for helping with the mice; and David Nadziejka for technical editing of the manuscript. The Van Andel Foundation supported this work.

\section{Author details}

${ }^{1}$ Center for Skeletal Disease and Tumor Metastasis, Van Andel Research Institute, 333 Bostwick Avenue NE, Grand Rapids, MI 49503, USA. ${ }^{2}$ Center for Cancer and Cell Biology, Van Andel Research Institute, 333 Bostwick Avenue NE, Grand Rapids, Ml 49503, USA. ${ }^{3}$ Michigan State University College of Human Medicine, 15 Michigan Street NE, Grand Rapids, Ml 49503, USA. ${ }^{4}$ Division of Pulmonary \& Critical Care Medicine, University of Michigan, Biomedical Science Research Building, 109 Zina Pitcher Place Room 4062, Ann Arbor, Ml 48109-2200, USA.

\section{Received: 20 October 2013 Accepted: 13 March 2014}

Published: 27 March 2014

\section{References}

1. Loeser RF, Goldring SR, Scanzello CR, Goldring MB: Osteoarthritis: a disease of the joint as an organ. Arthritis Rheum 2012, 64:1697-1707.

2. Lorenz H, Richter W: Osteoarthritis: cellular and molecular changes in degenerating cartilage. Prog Histochem Cytochem 2006, 40:135-163.

3. Goldring MB, Marcu KB: Cartilage homeostasis in health and rheumatic diseases. Arthritis Res Ther 2009, 11:224.

4. Borrelli J Jr, Silva MJ, Zaegel MA, Franz C, Sandell LJ: Single high-energy impact load causes posttraumatic $O A$ in young rabbits via a decrease in cellular metabolism. J Orthop Res 2009, 27:347-352.

5. Hashimoto S, Rai MF, Janiszak KL, Cheverud JM, Sandell LJ: Cartilage and bone changes during development of post-traumatic osteoarthritis in selected LGXSM recombinant inbred mice. Osteoarthritis Cartilage 2012, 20:562-571.

6. Sandell L: Etiology of osteoarthritis: genetics and synovial joint development. Nat Rev Rheumatol 2012, 8:77-89.

7. McGlashan SR, Cluett EC, Jensen CG, Poole CA: Primary cilia in osteoarthritic chondrocytes: from chondrons to clusters. Dev Dyn 2008, 237:2013-2020.

8. Scanzello CR, Goldring SR: The role of synovitis in osteoarthritis pathogenesis. Bone 2012, 51:249-257.
9. Gravallese EM, Goldring SR: Cellular mechanisms and the role of cytokines in bone erosions in rheumatoid arthritis. Arthritis Rheum 2000, 43:2143-2151.

10. Citri A, Yarden Y: EGF-ERBB signalling: towards the systems level. Nat Rev Mol Cell Biol 2006, 7:505-516.

11. Schneider MR, Sibilia M, Erben RG: The EGFR network in bone biology and pathology. Trends Endocrinol Metab 2009, 20:517-524.

12. Xian CJ: Roles of epidermal growth factor family in the regulation of postnatal somatic growth. Endocr Rev 2007, 28:284-296.

13. Zhang X, Tamasi J, Lu X, Zhu J, Chen H, Tian X, Lee TC, Threadgill DW, Kream BE, Kang Y, Partridge NC, Qin L: Epidermal growth factor receptor plays an anabolic role in bone metabolism in vivo. J Bone Miner Res 2011, 26:1022-1034

14. Zhang X, Siclari VA, Lan S, Zhu J, Koyama E, Dupuis HL, Enomoto-Iwamoto $M$, Beier F, Qin L: The critical role of the epidermal growth factor receptor in endochondral ossification. J Bone Miner Res 2011, 26:2622-2633.

15. Sibilia M, Wagner B, Hoebertz A, Elliott C, Marino S, Jochum W, Wagner EF: Mice humanised for the EGF receptor display hypomorphic phenotypes in skin, bone and heart. Development 2003, 130:4515-4525.

16. Usmani SE, Pest MA, Kim G, Ohora SN, Qin L, Beier F: Transforming growth factor a controls the transition from hypertrophic cartilage to bone during endochondral bone growth. Bone 2012, 51:131-141.

17. Lo SF, Wan L, Lin HC, Huang CM, Chen SY, Liu SC, Tsai FJ: Association of rheumatoid arthritis risk with EGFR genetic polymorphisms in Taiwan's Han Chinese population. Rheumatol Int 2012, 32:2301-2306.

18. Hallbeck AL, Walz TM, Briheim K, Wasteson A: TGF- $a$ and ErbB2 production in synovial joint tissue: increased expression in arthritic joints. Scand J Rheumatol 2005, 34:204-211.

19. Swanson CD, Akama-Garren EH, Stein EA, Petralia JD, Ruiz PJ, Edalati A, Lindstrom TM, Robinson WH: Inhibition of epidermal growth factor receptor tyrosine kinase ameliorates collagen-induced arthritis. J Immunol 2012, 188:3513-3521.

20. Sumariwalla PF, Jin P, Zhang J, Ni I, Crawford D, Shepard HM, Paleolog EM Feldmann M: Antagonism of the human epidermal growth factor receptor family controls disease severity in murine collagen-induced arthritis. Arthritis Rheum 2008, 58:3071-3080.

21. Appleton CT, Usmani SE, Mort JS, Beier F: Rho/ROCK and MEK/ERK activation by transforming growth factor- $a$ induces articular cartilage degradation. Lab Invest 2010, 90:20-30.

22. Appleton CT, Usmani SE, Bernier SM, Aigner T, Beier F: Transforming growth factor a suppression of articular chondrocyte phenotype and Sox9 expression in a rat model of osteoarthritis. Arthritis Rheum 2007, 56:3693-3705.

23. lacob S, CS-Szabo G: Biglycan regulates the expression of EGF receptors through EGF signaling pathways in human articular chondrocytes. Connect Tissue Res 2010, 51:347-358.

24. Zhang YW, Vande Woude GF: Mig-6, signal transduction, stress response and cancer. Cell Cycle 2007, 6:507-513.

25. Zhang X, Pickin KA, Bose R, Jura N, Cole PA, Kuriyan J: Inhibition of the EGF receptor by binding of MIG6 to an activating kinase domain interface. Nature 2007, 450:741-744.

26. Zhang YW, Su Y, Lanning N, Swiatek PJ, Bronson RT, Sigler R, Martin RW, Vande Woude GF: Targeted disruption of Mig-6 in the mouse genome leads to early onset degenerative joint disease. Proc Natl Acad Sci U S A 2005, 102:11740-11745.

27. Velasquillo C, Garciadiego-Cázares D, Almonte M, Bustamante M, Ibarra C, Kouri JB, Chimal-Monroy J: Expression of MIG-6, WNT-9A, and WNT-7B during osteoarthritis. Ann N Y Acad Sci 2007, 1117:175-180.

28. Louboutin H, Debarge R, Richou J, Selmi TA, Donell ST, Neyret P, Dubrana F: Osteoarthritis in patients with anterior cruciate ligament rupture: a review of risk factors. Knee 2009, 16:239-244.

29. Nelson F, Billinghurst RC, Pidoux I, Reiner A, Langworthy M, McDermott M, Malogne T, Sitler DF, Kilambi NR, Lenczner E, Poole AR: Early post-traumatic osteoarthritis-like changes in human articular cartilage following rupture of the anterior cruciate ligament. Osteoarthritis Cartilage 2006, 14:114-119.

30. Kuroki K, Cook CR, Cook JL: Subchondral bone changes in three different canine models of osteoarthritis. Osteoarthritis Cartilage 2011, 19:1142-1149.

31. Jin N, Cho SN, Raso MG, Wistuba I, Smith Y, Yang Y, Kurie JM, Yen R, Evans CM, Ludwig T, Jeong JW, DeMayo FJ: Mig-6 is required for appropriate lung development and to ensure normal adult lung homeostasis. Development 2009, 136:3347-3356. 
32. Kamekura S, Hoshi K, Shimoaka T, Chung U, Chikuda H, Yamada T, Uchida M, Ogata N, Seichi A, Nakamura K, Kawaguchi H: Osteoarthritis development in novel experimental mouse models induced by knee joint instability. Osteoarthritis Cartilage 2005, 13:632-641.

33. Pan J, Wang B, Li W, Zhou X, Scherr T, Yang Y, Price C, Wang L: Elevated cross-talk between subchondral bone and cartilage in osteoarthritic joints. Bone 2012, 51:212-217.

34. Joiner DM, Less KD, Van Wieren EM, Hess D, Williams BO: Heterozygosity for an inactivating mutation in low-density lipoprotein-related receptor 6 (Lrp6) increases osteoarthritis severity in mice after ligament and meniscus injury. Osteoarthritis Cartilage 2013, 21:1576-1585.

35. Pritzker KP, Gay S, Jimenez SA, Ostergaard K, Pelletier JP, Revell PA, Salter D, van den Berg WB: Osteoarthritis cartilage histopathology: grading and staging. Osteoarthritis Cartilage 2006, 14:13-29.

36. Yi T, Lee HL, Cha JH, Ko SI, Kim HJ, Shin HI, Woo KM, Ryoo HM, Kim GS, Baek JH: Epidermal growth factor receptor regulates osteoclast differentiation and survival through cross-talking with RANK signaling. J Cell Physio/ 2008, 217:409-422.

37. Bell NH: RANK ligand and the regulation of skeletal remodeling. $J$ Clin Invest 2003, 111:1120-1122.

38. Pante G, Thompson J, Lamballe F, Iwata T, Ferby I, Barr FA, Davies AM, Maina F, Klein R: Mitogen-inducible gene 6 is an endogenous inhibitor of HGF/Met-induced cell migration and neurite growth. J Cell Biol 2005, 171:337-348.

39. Rush JS, Quinalty LM, Engelman L, Sherry DM, Ceresa BP: Endosomal accumulation of the activated epidermal growth factor receptor (EGFR) induces apoptosis. J Biol Chem 2012, 287:712-722.

40. Bhowmik A, Das N, Pal U, Mandal M, Bhattacharya S, Sarkar M, Jaisankar P, Maiti NC, Ghosh MK: 2,2'-diphenyl-3,3'-diindolylmethane: a potent compound induces apoptosis in breast cancer cells by inhibiting EGFR pathway. PLoS One 2013, 8:e59798.

41. Tracy S, Mukohara T, Hansen M, Meyerson M, Johnson BE, Jänne PA: Gefitinib induces apoptosis in the EGFR ${ }^{L 58 R}$ non-small-cell lung cancer cell line H3255. Cancer Res 2004, 64:7241-7244.

42. Hopkins S, Linderoth E, Hantschel O, Suarez-Henriques P, Pilia G, Kendrick H, Smalley MJ, Superti-Furga G, Ferby I: Mig6 is a sensor of EGF receptor inactivation that directly activates c-Abl to induce apoptosis during epithelial homeostasis. Dev Cell 2012, 23:547-559.

43. Chen YC, Colvin ES, Maier BF, Mirmira RG, Fueger PT: Mitogen-inducible gene 6 triggers apoptosis and exacerbates ER stress-induced $\beta$-cell death. Mol Endocrinol 2013, 27:162-171.

44. Xu J, Keeton AB, Wu L, Franklin JL, Cao X, Messina JL: Gene 33 inhibits apoptosis of breast cancer cells and increases poly(ADP-ribose) polymerase expression. Breast Cancer Res Treat 2005, 91:207-215.

45. Amishima M, Munakata M, Nasuhara Y, Sato A, Takahashi T, Homma Y, Kawakami Y: Expression of epidermal growth factor and epidermal growth factor receptor immunoreactivity in the asthmatic human airway. Am J Respir Crit Care Med 1998, 157:1907-1912.

46. Hur GY, Lee SY, Lee SH, Kim SJ, Lee KJ, Jung JY, Lee EJ, Kang EH, Jung KH, Lee SY, Kim JH, Shin C, Shim JJ, In KH, Kang KH, Yoo SH: Potential use of an anticancer drug gefinitib, an EGFR inhibitor, on allergic airway inflammation. Exp Mol Med 2007, 39:367-375.

47. Makkinje A, Quinn DA, Chen A, Cadilla CL, Force T, Bonventre JV, Kyriakis JM: Gene 33/Mig-6, a transcriptionally inducible adapter protein that binds GTP-Cdc42 and activates SAPK/JNK. A potential marker transcript for chronic pathologic conditions, such as diabetic nephropathy: possible role in the response to persistent stress. J Biol Chem 2000, 275:17838-17847.

48. Mateescu RG, Todhunter RJ, Lust G, Burton-Wurster N: Increased MIG-6 mRNA transcripts in osteoarthritic cartilage. Biochem Biophys Res Commun 2005, 332:482-486.

49. Tapper JE, Funakoshi Y, Hariu M, Marchuk L, Thornton GM, Ronsky JL, Zernicke $R$, Shrive NG, Frank CB: ACL/MCL transection affects knee ligament insertion distance of healing and intact ligaments during gait in the ovine model. J Biomech 2009, 42:1825-1833.

50. Kreider JM, Goldstein SA: Trabecular bone mechanical properties in patients with fragility fractures. Clin Orthop Relat Res 2009, 467:1955-1963.
51. Turk HF, Monk JM, Fan YY, Callaway ES, Weeks B, Chapkin RS: Inhibitory effects of $\omega$-3 fatty acids on injury-induced epidermal growth factor receptor transactivation contribute to delayed wound healing. Am J Physiol Cell Physiol 2013, 304:C905-C917.

52. Goldring SR: The role of bone in osteoarthritis pathogenesis. Rheum Dis Clin North Am 2008, 34:561-571.

doi:10.1186/ar4522

Cite this article as: Joiner et al:: Accelerated and increased joint damage in young mice with global inactivation of mitogen-inducible gene 6 after ligament and meniscus injury. Arthritis Research \& Therapy 2014 16:R81.

\section{Submit your next manuscript to BioMed Central and take full advantage of:}

- Convenient online submission

- Thorough peer review

- No space constraints or color figure charges

- Immediate publication on acceptance

- Inclusion in PubMed, CAS, Scopus and Google Scholar

- Research which is freely available for redistribution

Submit your manuscript at www.biomedcentral.com/submit
() Biomed Central 